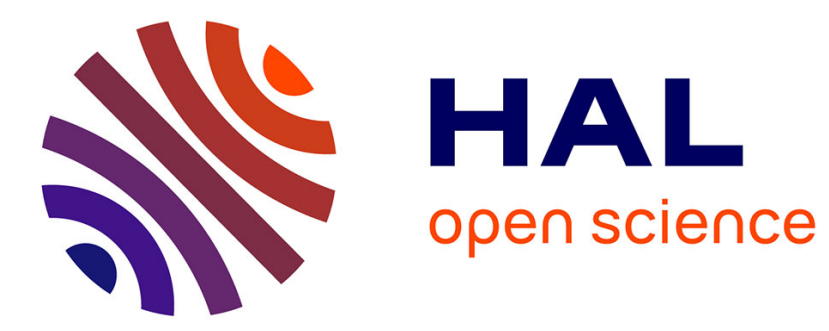

\title{
Electron spin resonance in heavily doped TMMC : Cu
}

\author{
S. Clément, C. Dupas, J.P. Renard, A. Cheikh-Rouhou
}

\section{To cite this version:}

S. Clément, C. Dupas, J.P. Renard, A. Cheikh-Rouhou. Electron spin resonance in heavily doped TMMC: Cu. Journal de Physique, 1982, 43 (5), pp.767-771. 10.1051/jphys:01982004305076700 . jpa-00209449

\section{HAL Id: jpa-00209449 https://hal.science/jpa-00209449}

Submitted on 1 Jan 1982

HAL is a multi-disciplinary open access archive for the deposit and dissemination of scientific research documents, whether they are published or not. The documents may come from teaching and research institutions in France or abroad, or from public or private research centers.
L'archive ouverte pluridisciplinaire HAL, est destinée au dépôt et à la diffusion de documents scientifiques de niveau recherche, publiés ou non, émanant des établissements d'enseignement et de recherche français ou étrangers, des laboratoires publics ou privés. 
Classification

Physics Abstracts

$76.30 \mathrm{~F}$

\title{
Electron spin resonance in heavily doped TMMC : $\mathbf{C u}$
}

\author{
S. Clément, C. Dupas, J. P. Renard \\ Institut d'Electronique Fondamentale (*), Université Paris-XI, Bât. 220, 91405 Orsay, France
}

and A. Cheikh-Rouhou

Faculté des Sciences et Techniques, Sfax, Tunisia.

(Reçu le 22 octobre 1981, accepté le 18 janvier 1982)

\begin{abstract}
Résumé. - Nous donnons les résultats de mesures de RPE dans le paramagnétique de Heisenberg unidimensionnel $\left(\mathrm{CH}_{3}\right)_{4} \mathrm{NMn}_{1-x} \mathrm{Cu}_{x} \mathrm{Cl}_{3}, x$ étant compris entre 0 et 0,5 . A température ambiante la largeur de raie est maximum pour une concentration $x \sim 0,25$ en accord avec le modèle de la diffusion freinée. On note cependant une exception pour l'orientation correspondant à l'angle magique. On observe un déplacement de la raie vers les bas champs dans les échantillons les plus dopés. La RPE est aussi modifiée par les impuretés aux basses températures. Les mesures sont en accord avec une réduction de la contribution gaussienne et une augmentation de la contribution diffusive.
\end{abstract}

\begin{abstract}
We report ESR measurements in the one-dimensional Heisenberg paramagnet $\left(\mathrm{CH}_{3}\right)_{4} \mathrm{NMn}_{1-x} \mathrm{Cu}_{x} \mathrm{Cl}_{3}$ for $x$ ranging between 0 and 0.5 . The room temperature results show a maximum of the linewidth for a concentration $x \sim 0.25$, in agreement with the altered diffusion model, with the notable exception of the magic angle orientation. A lineshift towards low fields is observed in strongly doped samples. At lower temperatures, ESR is also modified by the impurities, in accordance with the reduction of the short time effects and the enhancement of the diffusive contribution.
\end{abstract}

Since the pioneering work of Dietz et al. [1] much work has been done on the spin dynamics in low dimensional systems [2]. It is now well established that the decay of spin correlations functions in quasi-1d systems such as $\left(\mathrm{CH}_{3}\right)_{4} \mathrm{NMnCl}_{3}$ (TMMC) is very slow, being characterized by a long time diffusive behaviour, i.e. a $(D t)^{-1 / 2}$ law, where $D$ is the spin diffusion coefficient. The exchange narrowing of the ESR line is then much less effective than in $3 d$ and peculiar features of the spectrum may be observed.

Some interesting results have also been obtained for the case of impurity-doped linear chains. Since the excitation in the spin system cannot avoid the impurity placed in the chain, stronger effects than in two or three dimensional systems are expected. P. M. Richards [3] has reported that substitution of $\mathrm{Mn}^{++}$ ions by $\mathrm{Cu}^{++}$in TMMC results in an appreciable broadening of the linewidth without modification of the lineshape. Other dimensional effects such as shifts [4] and satellite lines [5] are also amplified by the doping. These effects can be explained by a slowing down of the rate of diffusion along the chains caused

(*) Laboratoire associé au C.N.R.S. by the $\mathrm{Cu}^{++}$ions. In a model described by Richards [3], the chains are cut into segments in which the spins diffuse at an unchanged rate $D(0)$ and others in which the diffusion coefficient is $D^{\prime}$, proportional to the $\mathrm{Mn}-\mathrm{Cu}$ exchange interaction. The effective spindiffusion coefficient at concentration $x, D(x)$, is then given by :

$$
\frac{1}{D(x)}=\frac{1-2 x}{D(0)}+\frac{2 x}{D^{\prime}}
$$

This model, valid for low values of $x(x<0.2)$ is well supported by the studies of the frequency dependence of proton relaxation rate [6] and ESR linewidth [7] in TMMC.

In this paper we report ESR measurements at room and low temperatures in heavily doped samples of $\left(\mathrm{CH}_{3}\right)_{4} \mathrm{NMn}_{1-x} \mathrm{Cu}_{x} \mathrm{Cl}_{3}$. Our results will be discussed in terms of a model extended to the case of arbitrary values of $x$. The crystals used in the present study were grown by slow evaporation of saturated solutions of $\left(\mathrm{CH}_{3}\right)_{4} \mathrm{NCl}$ and

$$
\left[y \mathrm{CuCl}_{2} .2 \mathrm{H}_{2} \mathrm{O}+(1-y) \mathrm{MnCl}_{2} \cdot 4 \mathrm{H}_{2} \mathrm{O}\right]
$$




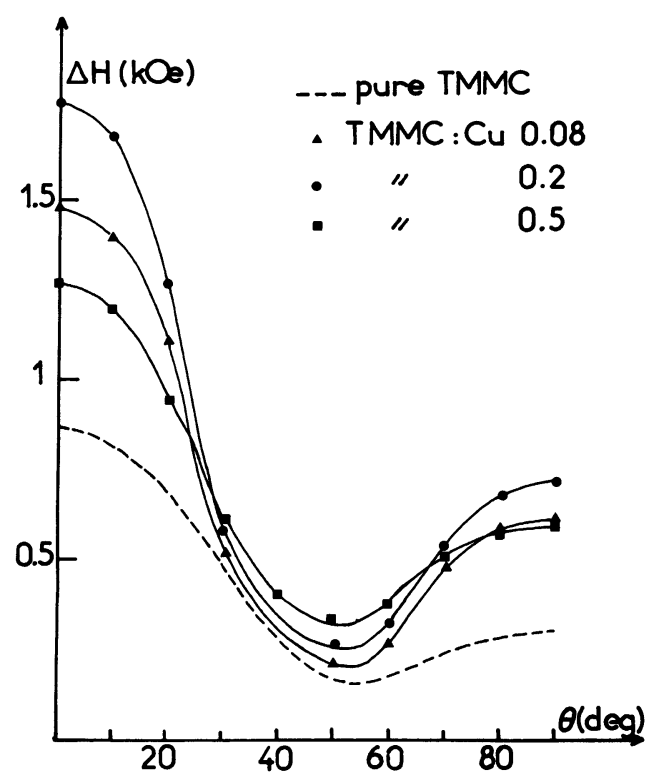

Fig. 1. - Angular dependence of the peak-to-peak derivative linewidth $\Delta H$ in TMMC : Cu at $300 \mathrm{~K} . \theta$ is the angle between the applied field $\mathbf{H}_{0}$ and the chain axis $\mathrm{c}$. The lines are only guides to the eye.

in concentrated hydrochloric acid at about $50^{\circ} \mathrm{C}$. The growing of single crystals was not easy but we succeeded in obtaining $\mathrm{Cu}^{++}$concentrations as high as $x=0.5$. However good samples of pure TMCuC $(x=1)$ could not be obtained. The $\mathrm{Mn}$ and $\mathrm{Cu}$ concentrations of the samples were determined by the Service Central d'Analyse du C.N.R.S. Measurements

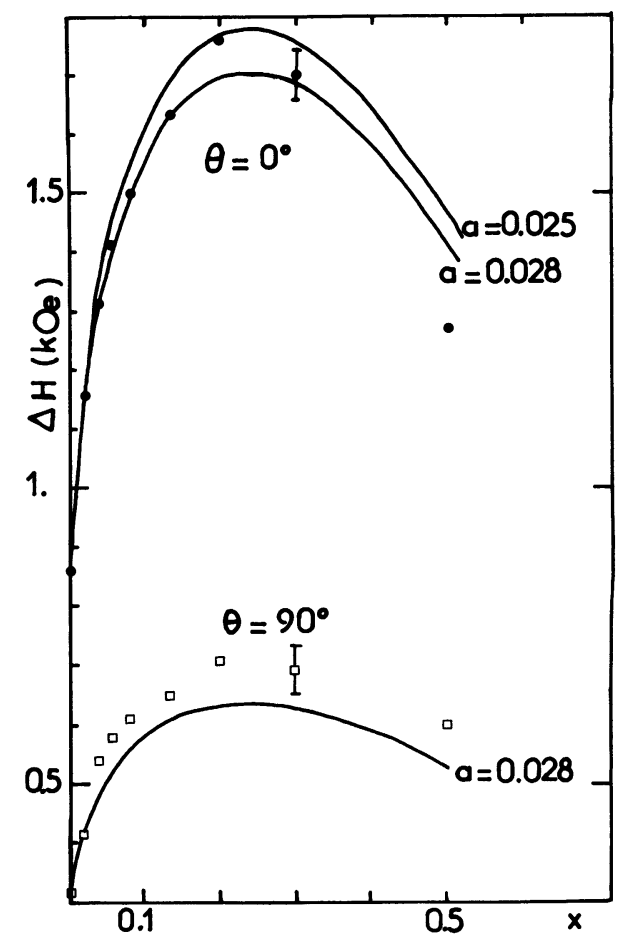

Fig. 2. - Variation of the linewidth $\Delta H$ vs. Cu concentration $x$ in TMMC : $\mathrm{Cu}$ at $300 \mathrm{~K}$. Two cases are considered : $\mathbf{H}_{0} / / \mathbf{c}\left(\theta=0^{\circ}\right)$ and $\mathbf{H}_{0} \perp \mathbf{c}\left(\theta=90^{\circ}\right)$. The theoretical curves are based on equation (3). of the ESR absorption derivative were performed in a conventional spectrometer operating at $9.3 \mathrm{GHz}$.

1. Room temperature results. - We first discuss the room temperature results. Figure 1 shows the angular and $\mathrm{Cu}$-concentration dependence of the peak-topeak derivative linewidth $\Delta H . \theta$ is the angle between the chain axis and the applied field. We focus especially on three orientations : $\theta=0^{\circ}$ and $\theta=90^{\circ}$ (Fig. 2) and the "magic angle " $\theta=55^{\circ}$ (Fig. 3). The salient feature for the first two orientations is the increase of the linewidth as the concentration $x$ is rising, until a maximum is reached at $x \simeq 0.25$.

Theoretical treatments of the ESR lineshape in 1d systems have been given by several authors $[8,9]$. The dominant broadening mechanism is the dipole-dipole interaction between spins along the chain. The profile of the resonance is related to the so-called « total-spintorque " (TST) correlation function which involves time dependent correlations between dipolar terms. It is convenient to separate this function into a Gaussian short time part and a diffusive part [9]. At room temperature this latter part is usually largely dominant. Furthermore, when $\theta=0^{\circ}$, only the secular term of the dipolar interaction is involved and we have then a typical $1 \mathrm{~d}$ effect : the line is non-lorentzian and

$$
\Delta H \propto\left[M_{2} D^{-1 / 2}\right]^{2 / 3}
$$

where $\mathrm{M}_{2}$ is the corresponding second moment of the line. Two effects result from the doping :

(i) a reduction of the second moment by a factor $(1-x)$ since the $\mathrm{Mn}^{++}$ions are replaced by $\mathrm{Cu}^{++}$ ions with much lower magnetic moments;

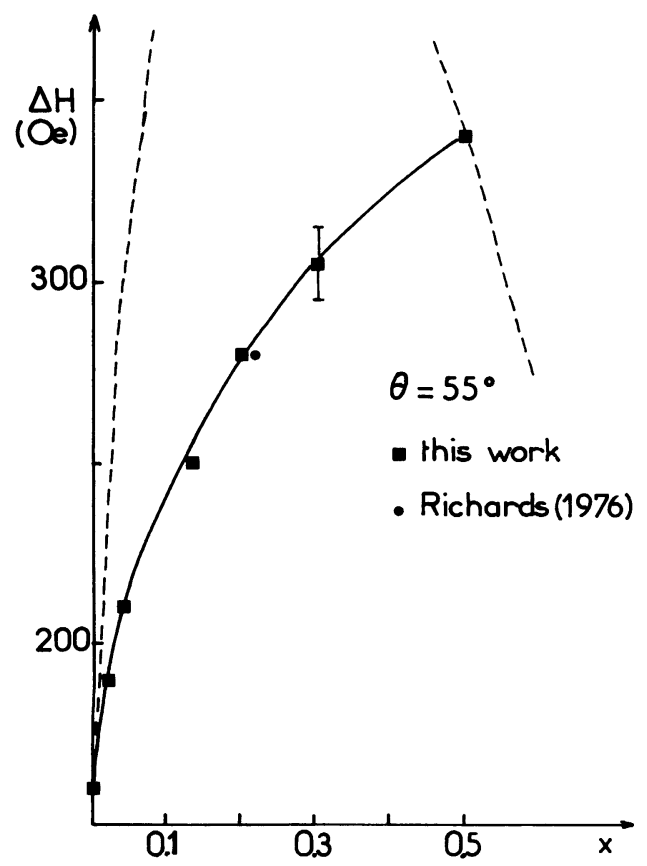

Fig. 3. - Variation of the linewidth $\Delta H$ vs. Cu concentration in TMMC : $\mathrm{Cu}$ at $300 \mathrm{~K}$ at the magic angle $\left(\theta=55^{\circ}\right)$. The full line is a guide to the eye. The dashed line is based on equation (4). 
(ii) following Richards' model, a slower diffusion process characterized by $D(x)$. If we suppose that the impurities are randomly distributed in the lattice, we get the following equation, valid for an arbitrary value of $x$ :

$$
\frac{1}{D(x)}=\frac{(1-x)^{2}}{D_{\mathrm{Mn}}}+\frac{2 x(1-x)}{D^{\prime}}+\frac{x^{2}}{D_{\mathrm{Cu}}} .
$$

This agrees with the relation (1) for $x \ll 1$.

Here we have $D_{\mathrm{Mn}} \simeq 2.66 J_{\mathrm{Mn}}[S(S+1)]^{1 / 2}[10]$ with $J_{\mathrm{Mn}}=6.7 \mathrm{~K}$ and $S=5 / 2$ while $D_{\mathrm{Cu}} \simeq 2.66 J_{\mathrm{Cu}} \times$ $[s(s+1)]^{1 / 2}$ with the exchange between $\mathrm{Cu}^{++}$ions $J_{\mathrm{Cu}}=45 \mathrm{~K}$ [11] and $s=1 / 2$. However we do not have, to date, precise knowledge of the relation between $D^{\prime}$ and the $\mathrm{Mn}-\mathrm{Cu}$ exchange. So we express $D^{\prime}$ as $D^{\prime}=a D_{M n}, a$ being the only adjustable parameter. We thus obtain, for the linewidth at concentration $x$ :

$$
\begin{aligned}
\Delta H(x)= & \Delta H(0)(1-x)^{2 / 3} \times \\
& {\left[x^{2}\left(1.5-\frac{2}{a}\right)-2 x\left(1-\frac{1}{a}\right)+1\right]^{1 / 3} . }
\end{aligned}
$$

Such a relation holds also approximately for the orientation $\theta=90^{\circ}$, for which the secular term of the dipolar interaction is dominant. In figure 2 we plot the corresponding theoretical curves and obtain a fairly good agreement with experiment with the value $a=0.028$. If we adopt the most simple relation for $D^{\prime}[3]: D^{\prime} \simeq 2.66 J_{\mathrm{Mn}-\mathrm{Cu}}[S(S+1) s(s+1)]^{1 / 4}$ this would lead to $J_{\mathrm{Mn}-\mathrm{Cu}} \simeq 0.4 \mathrm{~K}$, in good agreement with the results of Richards, but much lower than the value obtained from magnetic susceptibility and Néel temperature measurements $(1.4 \mathrm{~K})$ [12]. It should be noted, however, that the $x$ value corresponding to the maximum of the linewidth is nearly independent of the precise value of $a$, as long as $a \ll 1$; this is the case here, and the experimental agreement justifies in some ways the model used (eq. 2) irrespective of the $D^{\prime}$ value. We turn now to the "magic angle case" this orientation, only the non-secular terms of the dipolar interaction are concerned and the line is Lorentzian. On the basis of the model, we expect a variation

$$
\frac{\Delta H(x)}{\Delta H(0)}=(1-x)\left(\frac{D(0)}{D(x)}\right)^{1 / 2}
$$

presenting also a maximum near $x=0.25$. The experimental results (Fig. 3) are in complete disagreement with such an evolution : the linewidth increases monotonically, and less than expected. One can suspect the validity of the model for this particular case : the linewidth, here, is related to high frequency (the Larmor frequency $\omega_{z}$ and $2 \omega_{z}$ ) Fourier transforms of the TST function. A diffusive process is probed only if $\omega_{z}$ is much smaller than the diffusion coefficient. The condition $\omega_{z} \ll D^{\prime}$ is not entirely met here and a reduction of the enhancement can result. Some other effects should probably interfere, such as the decrease with $x$ of the contribution of the short time part of the TST function which is not negligible for this orientation. We have assumed, also, for simplicity, that only the spins of $\mathrm{Mn}^{2+}$ participate to the observed resonance. We have in fact two different types of spins with different $g$-values, which give a single resonance line since the $\mathrm{Mn}-\mathrm{Cu}$ exchange interaction is much greater than the difference in Zeeman energy of the ions. In a complete study, we should take into account these two sets of resonant spins with their interactions. The corrections to be added are expected to be relatively more important for the narrower magic angle line. It is interesting to point out that a Lorentzian shape is observed, at $\theta=55^{\circ}$, even in the most doped sample $(x=0.5)$.

One of the related effects is the $g$ factor variation which goes from $g_{\mathrm{Mn}} \simeq 2.0(x=0)$ to anisotropic $g_{\mathrm{Cu}}($ for $x=1)$.

A full description of the lineshifts in pure TMMC has not yet been given. We can distinguish a static shift due to the average dipolar field, which is quite small at room temperature, a dynamic dipolar shift $[8$, 9], related to the imaginary part of the TST function, and a "polarization" shift [4] which depends on the angle between the linearly polarized microwave field and the plane containing the chain axis and the static field. These last two contributions do depend on the rate of decay of spin correlations and are greatly enhanced by the doping [4] : the shift is approximately doubled for $x=0.045$. We have plotted on the figure 4

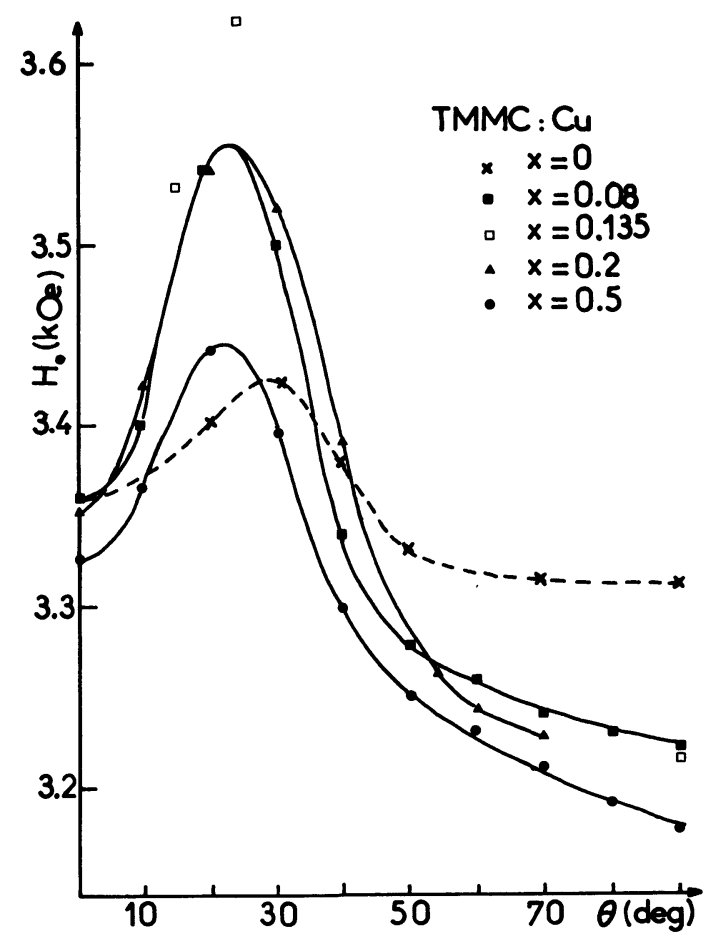

Fig. 4. - Angular dependence of the ESR field $H_{0}$ in TMMC : $\mathrm{Cu}$ at $T=300 \mathrm{~K}$. The lines are only guides to the eye. 
the resonance field of doped TMMC at room temperature versus the angle $\theta$ for several concentrations of impurities. We note an apparent maximum of the effect and a more evident displacement of the whole towards low fields for $x=0.50$. This behaviour is probably related to the increase of the $g$-value with $x$. However the negative shift, which exceeds a hundred Gauss for some orientations, corresponds to $g$ variations notably greater than those theoretically expected [13]. The noticeable positive shift observed when $\theta \sim 30^{\circ}$ is correlated with an asymmetry of the ESR line.

2. Low temperature linewidth. - The low temperature behaviour of the linewidth in 1d systems has been described by Tazuke and Nagata [14] and more recently by Cheung et al. [9]. Two competing processes must be considered : the diffusive part of the TST function decreases with decreasing temperature while the Gaussian part increases. As a result, a minimum may appear in the temperature dependence of the linewidth at moderately high temperatures; at lower temperatures the Gaussian part dominates strongly and is asymptotically proportional to $T^{-3}$.

Experimental results in pure TMMC $[9,15]$ are in good agreement with this model though one does not observe the minimum but rather a slow increase of the linewidth in the intermediate regime. We have measured the linewidth variation versus temperature in three copper doped samples $(x=0.08,0.20$ and 0.50$)$. The data obtained are shown in figure 5. Three particular orientations were considered, respectively $\theta=0^{\circ}$ (Fig. 5a), $\theta=55^{\circ}$ (Fig. $5 b$ ) and $\theta=90^{\circ}$ (Fig. 5c).

As the doping reduces the Gaussian contribution but enhances the diffusive part, one can predict a steep decrease of the linewidth at " high » temperatures and a less marked increase at "low" temperatures when $x$ is increased. Our results are in qualitative agreement with these predictions. In the $x=0.08$ sample the linewidth again seems to diverge, but at lower temperatures than in the pure sample. The minimum also is shifted towards low temperatures when $x$ increases. This behaviour can be compared to that observed recently [16] in dilute $2 \mathrm{~d} \mathrm{Rb}_{2} \mathrm{Mn}_{x} \mathrm{Mg}_{1-x} \mathrm{~F}_{4}$ for which the same arguments could be applied.

In conclusion, our results in heavily $\mathrm{Cu}$-doped TMMC are, in general, consistent with the predictions of the theory of ESR in 1d antiferromagnets combined with the model of slowing down of the diffusion by the impurities. A confirmation is given in particular by the linewidth maximum observed when the concentration is varied. However the magic angle results necessitate more complete explanations.

The temperature and concentration dependences of the resonance linewidth appear to be, as a whole, in qualitative agreement with existing theories. However a detailed quantitative comparison was not attempted here.
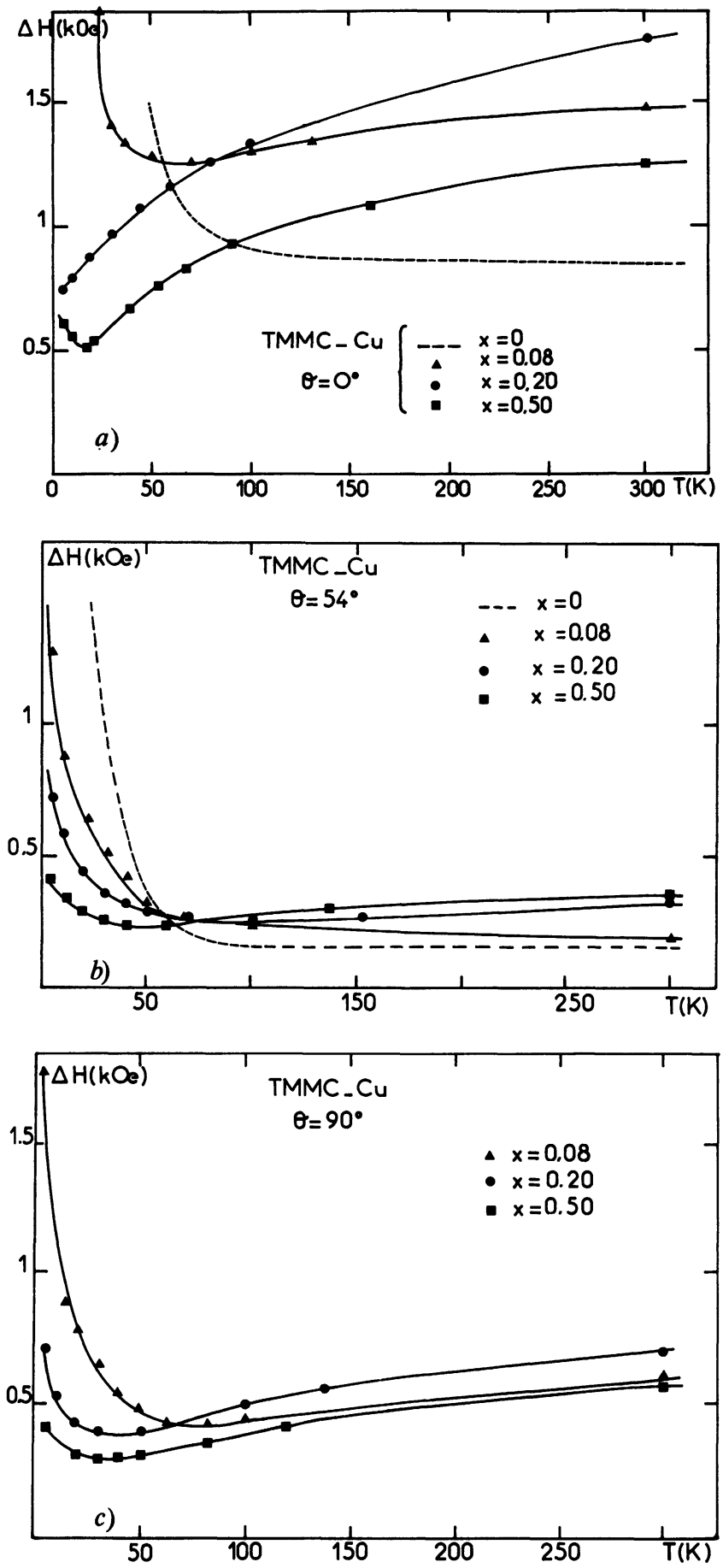

Fig. 5. - ESR linewidth of TMMC : Cu vs. temperature for the orientations : (a) $\theta=0^{\circ} ;(b) \theta=55^{\circ} ;$ (c) $\theta=90^{\circ}$. The rise of the linewidth appears at lower temperatures when the $\mathrm{Cu}$ concentration $x$ is increased. The $x=0$ results are taken from reference [15]. The full lines are guides to the eye.

Acknowledgments. - The ESR experiments reported here have been performed with the apparatus of Dr. P. Monod (Laboratoire de Physique des Solides, Bât. 510, University Paris XI-Orsay). The authors wish to thank Dr. Monod for his kind hospitality and for helpful discussions. 


\section{References}

[1] Dietz, R. E., Merritt, F. R., Dingle, R., Hone, D., Silbernagel, B. G. and Richards, P. M., Phys. Rev. Lett. 26 (1971) 1186.

[2] Steiner, M., Villain, J. and Windsor, C. G., Adv. Phys. 25 (1976) 87.

[3] Richards, P. M., Phys. Rev. B 10 (1974) 805, Phys. Rev. B 13 (1976) 458.

[4] Yamada, I. and Natsume, Y., J. Phys. Soc. Japan 48 (1980) 58.

[5] Komatsubara, T., Iio, K. and Nagata, K., J. Phys. Soc. Japan 45 (1978) 351.

[6] Clément, S. and Tchao, Y. H., C.R. Hebd. Séan. Acad. Sci. 282B (1976) 165.

[7] IbrügGer, J., Siegel, E. and LAGendiJK, A., J. Mag. Mag. Mater. 13 (1979) 107.

[8] Boucher, J. P., Ahmed-Bakheit, M. and NechtSChein, M., Villa, M., Bonera, G. and Borsa, F., Phys. Rev. B 13 (1976) 4098.

LagendiJk, A., Phys. Rev. B 18 (1978) 1322.
[9] Cheung, T. T. P., Soos, Z. G., Dietz, R. E. and MerRITT, F. R., Phys. Rev. B 17 (1978) 1266.

[10] Lurie, N. A., Huber, D. L. and Blume, M., Phys. Rev. B 9 (1974) 2171.

[11] Dupas, C., Renard, J. P., Seiden, J. and CheikhRouhou, A., Phys. Rev. B 25 (1982).

[12] Richards, P. M., Phys. Rev. B 14 (1976) 1239 ; Dupas, C. and Renard, J. P., Phys. Rev. B 18 (1978) 401.

[13] Huber, D. L., Phys. Rev. B 12 (1975) 31.

[14] Tazuke, Y. and Nagata, K., J. Phys. Soc. Japan. 38 (1975) 1003.

[15] Ablart, G., Bourdel, D., Pescia, J., Clément, S. and Renard, J. P., J. Physique Lett. 42 (1981) L-219.

[16] Walsh Jr, W. M., Birgeneau, R. J., Rupp Jr, L. W. and Guggenheim, H. J., Phys. Rev. B 20 (1979) 4645 . 